\title{
Role of transoesophageal echocardiography in evaluation of cardiogenic embolism
}

\author{
I W Black, A P Hopkins, L C L Lee, B M Jacobson, W F Walsh
}

\begin{abstract}
Objective-To determine the value of transoesophageal echocardiography in the assessment of selected patients at risk of cardiogenic embolism or after it.

Design-Prospective comparison of the results of transoesophageal and transthoracic echocardiography. Transoesophageal echocardiography was performed with a $5 \mathrm{MHz}$ single plane phased array transducer.

Setting-University teaching hospital.

Patients-100 patients referred for transoesophageal echocardiography after a cerebral ischaemic event or peripheral arterial embolism $(n=63)$, before percutaneous balloon dilatation of the mitral valve $(n=23)$, or before electrical cardioversion of atrial fibrillation ( $\mathrm{n}=14)$.
\end{abstract}

Results-Transthoracic echocardiography showed potential sources of embolism in four patients including left ventricular thrombus in two patients (with one false positive), left atrial appendage thrombus $(n=1)$, and patent foramen ovale $(n=1)$. Transoesophageal echocardiography showed 59 potential embolic sources in 45 patients including left atrial spontaneous echo contrast $(n=33)$, left atrial appendage thrombus $(n=13)$, left ventricular thrombus $(n=5)$, patent foramen ovale $(\mathbf{n}=3)$, left ventricular spontaneous echo contrast $(n=2)$, mitral valve prosthesis thrombus $(n=1)$, mitral valve prolapse $(n=1)$, and pronounced aortic atheroma $(n=1)$. Transoesophagal echocardiography showed potential embolic sources in $36 / 53(68 \%)$ patients with atrial fibrillation compared with $9 / 47(19 \%)$ patients in sinus rhythm. Percutaneous balloon dilatation of the mitral valve was performed without embolic complications in 18 patients without left atrial thrombi and in three patients with small fixed thrombi in the left atrial appendage. It was cancelled in two patients with large thrombi in the left atrial appendage. Cardioversion was performed without embolic complications in 14 patients without left atrial thrombi.

Conclusions-Transoesophageal echocardiography detects potential sources of embolism better than transthoracic echocardiography in selected patients at risk of cardiogenic embolism or after it.
Cardiogenic embolism accounts for $15 \%$ of ischaemic strokes and transient ischaemic attacks ${ }^{12}$ and is an important cause of systemic arterial embolism. ${ }^{3}$ Cardiogenic embolism can also complicate therapeutic procedures, including percutaneous balloon dilatation of the mitral valve ${ }^{4}$ and cardioversion of atrial fibrillation. ${ }^{5}$

Transthoracic echocardiography has been used to detect intracardiac thrombi and other potential cardiac sources of embolism. ${ }^{67}$ Recently the use of transoesophageal echocardiography to detect left atrial thrombi and other cardiac sources of embolism has been reported..$^{8-11}$ This technique provides high quality imaging of the cardiac anatomy including the left atrial body and appendage, which are close to the oesophagus, without interference from the chest wall and lungs. ${ }^{12}$

In a preliminary report in patients with a history of embolism, transoesophageal echocardiography was found to be more sensitive than transthoracic echocardiography for the detection of left atrial thrombi and also of left atrial spontaneous echo contrast. ${ }^{10}$ The latter term refers to swirling, smoke-like echoes seen in the left atrial cavity in patients with relative stasis of left atrial blood, ${ }^{13}$ and this feature was reported to be an independent predictor of thromboembolic risk in patients with mitral valve disease. ${ }^{14}$ Furthermore, there is little information about the role of transoesophageal echocardiography in patients undergoing procedures with a risk of cardiogenic embolism, such as electrical cardioversion of atrial fibrillation and percutaneous balloon dilatation of the mitral valve.

We prospectively assessed the overall role of transoesophageal echocardiography in the detection of potential cardiac sources of embolism by comparing the results of transoesophageal with those of transthoracic echocardiography both in patients referred after suspected cardiogenic embolism and in patients before procedures with a risk of cardiogenic embolism.

\section{Patients and methods}

PATIENTS

Between October 1988 and April 1990, 100 patients (62 women and 38 men, mean age 60 (range 25-86)) were referred for transoesophageal echocardiography after suspected cardiogenic embolism $(n=63)$, before percutaneous balloon dilatation of the mitral valve $(n=23)$, or before DC cardioversion of atrial fibrillation $(n=14)$. 
Sixty three patients were referred for the detection of potential cardiac sources of emboli after a cerebral ischaemic event or acute peripheral arterial occlusion. Patients were selected for transoesophageal echocardiography if cardiogenic embolism was clinically suspected and if transthoracic echocardiography alone was considered unlikely to detect potential embolic sources. Studies were performed within one month of the event in $59 / 63(94 \%)$ patients. Fifty two patients were studied after stroke or transient ischaemic attack. Cerebral infarction was shown by computed tomography or magnetic resonance imaging in $35 / 52(67 \%)$ patients. The remaining 11 patients were studied after acute thromboembolism to the arm or leg, documented at surgery in 10/11 patients. Two patients referred after suspected embolism had mitral stenosis and six patients had a mitral valve prosthesis.

Twenty three patients were studied before elective percutaneous balloon dilatation of the mitral valve. Transoesophageal echocardiography was routinely performed one day before dilatation to assess mitral valve anatomy and function and to detect left atrial thrombi.

Fourteen patients were referred to exclude left atrial thrombus before elective $\mathrm{DC}$ cardioversion of atrial fibrillation. Atrial fibrillation had lasted less than one month in seven patients and more than one month in seven patients.

Transoesophageal echocardiography was unsuccessful in two additional patients, referred after stroke, who were excluded from further analysis. Oesophageal intubation was unsuccessful in one patient and the second patient was unable to tolerate the endoscope despite apparently adequate sedation.

Twenty one of the 100 patients also underwent 26 follow up studies; 16 studies to assess the mitral valve apparatus and to detect possible iatrogenic atrial septal defects after percutaneous balloon dilatation of the mitral valve, and 10 studies to assess the effect of anticoagulant therapy on cardiac thrombi. One patient refused follow up study.

\section{METHODS}

All patients had initial conventional cross sectional and Doppler transthoracic echocardiography, including colour flow mapping, with $1.9 \mathrm{MHz}, 2.5 \mathrm{MHz}$, and 3.5 MHz transducers (Hewlett Packard 77020A). Mitral valve area

Table 1 Echocardiographic findings

\begin{tabular}{lll}
\hline & Transthoracic & Transoesophageal \\
\hline Normal & 96 & 55 \\
Left atrial SEC & 0 & 33 \\
Left atrial appendage thrombus & 1 & 13 \\
Left ventricular thrombus & 2 & 5 \\
Patent foramen ovale & 1 & 3 \\
Left ventricular SEC & 0 & 2 \\
Mitral valve prolapse & 0 & 1 \\
Mitral prosthesis thrombus & 0 & 1 \\
Aortic atheroma & 0 & 1
\end{tabular}

SEC, spontaneous echo contrast. was calculated by the Doppler pressure halftime method. ${ }^{15}$

Transoesophageal echocardiography was performed with a standard $5 \mathrm{MHz}$ single plane phased array transducer (Hewlett Packard 21236A). Written informed consent was obtained and the patients were examined in the fasting state. Intravenous sedation (midazolam with fentanyl) was given in 93/ $126(74 \%)$ of studies. Previously described tomographic planes, ${ }^{12}$ including basal short axis, four chamber, transgastric short axis, and views of the thoracic aorta were obtained. Particular attention was directed to the left atrial body and appendage and the interatrial septum. The left ventricular endocardium was examined in sequential transgastric short axis views as well as in four-chamber views.

Intracardiac thrombus was diagnosed by the presence of a clearly defined echodense intracavitary mass, acoustically distinct from the underlying endocardium and not caused by pectinate ridges of the atrial appendage. Mital valve prolapse was diagnosed by transoesophageal echocardiography according to previously reported criteria. ${ }^{9}$ From November 1989, contrast studies to detect a patient foramen ovale were performed during transoesophageal echocardiography in patients in whom no other source of embolism had been found $(n=25)$. Agitated saline containing air microbubbles was injected rapidly into an antecubital vein and the patient was instructed to perform a Valsalva manoeuvre or to cough. ${ }^{16}$ A patent foramen ovale with right to left shunting was diagnosed if microbubbles were seem to cross the atrial septum into the left atrium and no septal defect was seen.

The study period lasted 10-20 minutes and technically adequate images were obtained in all studies. There were no complications. All clinical and echocardiographic data were recorded prospectively. Transoesophageal and transthoracic echocardiographic studies were performed and interpreted independently by different experienced echocardiographers, who were, however, not blinded to the transthoracic echocardiography results.

Differences in proportions were compared by the $\chi^{2}$ test with continuity correction. Statistical significance was defined as $p<0.05$.

\section{Results}

Table 1 summarises echocardiographic findings in the 100 initial studies. Transoesophageal echocardiography was significantly more sensitive than transthoracic echocardiography in the detection of left atrial appendage thrombus $(p<0.005)$ and of left atrial spontaneous echo contrast $(p<0.0001)$. In the 47 patients in sinus rhythm, transoesophageal echocardiography showed potential embolic sources in nine $(19 \%)$ patients whereas thoracic echocardiography showed them in three patients $(6 \%)(p=N S)$. In the 53 patients with atrial fibrillation,transoesophagealechocardiography showed potential embolic sources in 36 patients $(68 \%)$ and transthoracic echocardiography showed them in one patient $(2 \%)(p<0.0001)$. 


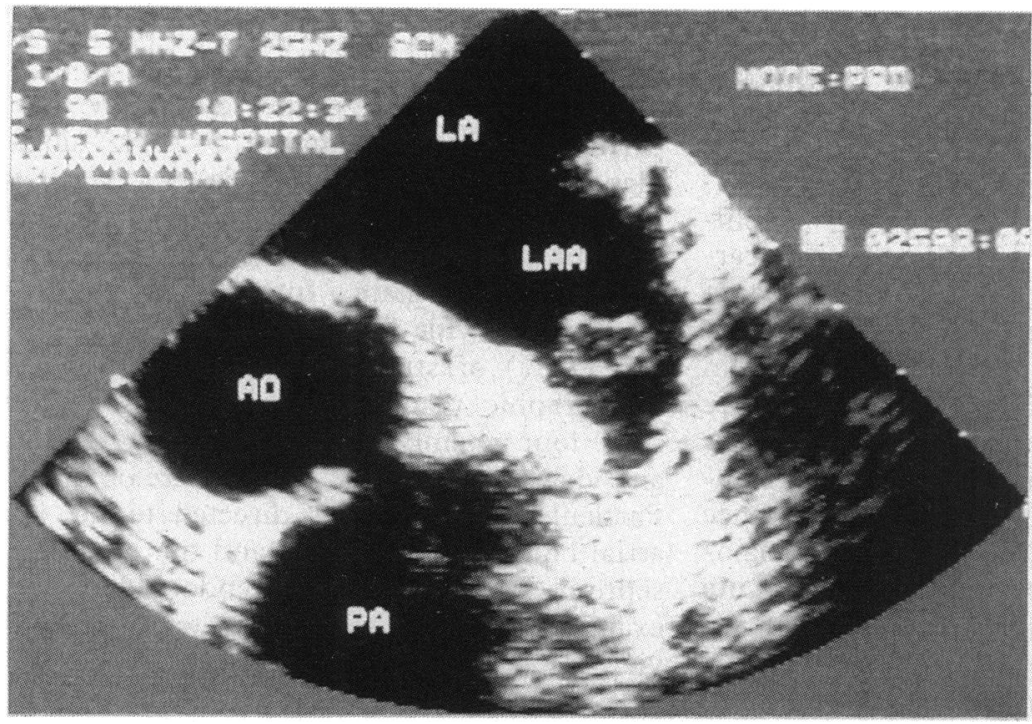

Figure 1 Thrombus in the left atrial appendage of a patient with atrial fibrillation and recent stroke. $L A$, left atrium; $L A A$, left atrial appendage; $A O$, aorta; $P A$, pulmonary artery. a patent foramen ovale. One patient had posterior leaflet mitral valve prolapse not clearly demonstrated by transthoracic echocardiography. One patient with mitral stenosis and left atrial enlargement ( $M$ mode dimension $52 \mathrm{~mm}$ ) was shown to have a left atrial appendage thrombus.

None of the 27 patients with atrial fibrillation had potential embolic sources shown by transthoracic echocardiography compared with $21 / 27(78 \%)$ patients in whom they were shown by transoesophageal echocardiography. Six patients had a thrombus in the left atrial appendage (fig 1) associated with left atrial spontaneous echo contrast in four patients. Eleven patients had left atrial spontaneous echo contrast without thrombus, including one patient with associated left ventricular spontaneous echo contrast and one with a patent foramen ovale. Two patients had small left ventricular thrombi, one patient without evidence of endocarditis had echoes consistent with thrombus on a mechanical mitral valve prosthesis, and one patient with embolism to the leg had considerable atheroma of the descending thoracic aorta.

Transoesophageal echocardiography showed potential sources of embolism in $22 / 52$ patients with cerebral ischaemic events and in 5/11 patients with acute peripheral arterial occlusion $(p=N S)$. Left atrial thrombi were found in one of two patients with mitral stenosis, one of six patients with mitral valve prostheses, and 5/22 patients with non-valvar atrial fibrillation. All five patients with left ventricular thrombus had previous anterior myocardial infarction. Eleven of the 13 patients found to have cardiac thrombi were receiving anticoagulant therapy at the time of study, and after the finding of left atrial appendage thrombus anticoagulant therapy was started in two patients not previously treated.

Four patients with thrombus at initial transoesophageal echocardiography underwent eight follow up studies after the start of warfarin treatment. Left atrial appendage thrombi were unchanged in two patients studied serially up to nine and 13 months. A left atrial appendage thrombus was found to have resolved in one patient after eight months. A left ventricular thrombus was found to have resolved at five months in one patient.

\section{PERCUTANEOUS BALLOON DILATATION OF THE} MITRAL VALVE

Eleven of the 23 patients were in sinus rhythm and 12 were in atrial fibrillation. In the 11 patients in sinus rhythm (mean mitral valve area $1.0 \mathrm{~cm}^{1}$ ) neither echocardiographic study showed thrombus while transoesophageal echocardiography alone showed left atrial spontaneous echo contrast in three patients.

In 12 patients with atrial fibrillation (mean mitral valve area $1.2 \mathrm{~cm}^{2}$ ) transthoracic echocardiography showed a large left atrial appendage thrombus in only one patient, which was confirmed by transoesophageal echocardiography and subsequent surgery. In the remaining 11 patients, left atrial thrombus was shown by transoesophageal echocardiography in five,
Figure 2 Dense left atrial spontanenus ticho contrast in a patient with atrial fibrillation and mitral stenosis. $L A$, left atrium; $L . V$, left ventricle; $R V$, right ventricle. 
Table 2 Effect of percutaneous balloon dilatation of the mitral valve on left atrial spontaneous echo contrast (SEC)

\begin{tabular}{|c|c|c|c|c|}
\hline \multirow{2}{*}{\multicolumn{2}{|c|}{ Group }} & \multirow[b]{2}{*}{$n(A F)$} & \multicolumn{2}{|c|}{ Mitral valve area } \\
\hline & & & Pre dilatation & Post dilatation \\
\hline $\begin{array}{l}\text { (I) } \\
\text { (II) } \\
\text { (III) }\end{array}$ & $\begin{array}{l}\text { No SEC in either study } \\
\text { Persistence of SEC } \\
\text { Resolution of SEC }\end{array}$ & $\begin{array}{ll}6 & (1) \\
7 & (6) \\
3 & (1)\end{array}$ & $\begin{array}{ll}1.3 & (1.0-1.9) \\
1.1 & (0.9-1.4) \\
0.9 & (0.8-1.2)\end{array}$ & $\begin{array}{ll}1.6 & (1 \cdot 0-2 \cdot 2) \\
1.5 & (1 \cdot 4-1.9) \\
2 \cdot 2 & (2 \cdot 0-2 \cdot 4)\end{array}$ \\
\hline
\end{tabular}

Mitral valve mean area and range are shown. AF, atrial fibrillation.

all in the appendage and all associated with left atrial spontaneous echo contrast. Five patients had left atrial spontaneous echo contrast alone (fig 2). Only one patient had neither thrombus nor spontaneous contrast.

Balloon dilatation of the mitral valve was not performed in three of the six patients with thrombus, all with large thrombi in the atrial appendage. One patient was treated medically and the second patient underwent mitral valve replacement at which the presence of a left atrial appendage thrombus was confirmed. Warfarin was started in the third patient who underwent successful percutaneous balloon dilatation of the mitral valve six months later after repeat transoesophageal echocardiography showed complete resolution of the thrombus. Three other patients had small fixed thrombi at the apex of the left atrial appendage. Percutaneous balloon dilatation of the mitral valve was performed in these patients with the Inoue balloon technique ${ }^{17}$ without embolic or other complication. All 18 patients without evidence of left atrial thrombus underwent balloon dilatation without embolic complications.

Sixteen patients had follow up transoesophageal echocardiography three months after valve dilatation. Table 2 shows the effect of valve dilatation on left atrial spontaneous echo contrast. In three patients with left atrial appendage thrombus at initial study, the thrombi had resolved at follow up study in one patient with persistence of spontaneous contrast and in one patient with resolution of spontaneous contrast. One patient with resolution of spontaneous contrast had partial resolution of thrombus.

One patient in chronic atrial fibrillation and little change in mitral valve area, from $1.3 \mathrm{~cm}^{2}$ to $1.5 \mathrm{~cm}^{2}$, was found to have developed thrombus in the body of the left atrium at follow up, in addition to the persistence of spontaneous echo contrast. A third study six weeks later after the start of warfarin showed complete resolution of thrombus.

\section{CARDIOVERSION}

Transthoracic echocardiography was negative in all patients. Four patients were shown to have left atrial spontaneous echo contrast by transoesophageal echocardiography, including both patients with mitral prostheses and one of three patients with mitral stenosis. Only one of nine patients with non-valvar atrial fibrillation had left atrial spontaneous echo contrast. No patient had left atrial thrombus. Cardioversion was performed within 24 hours of echocardiography in all patients and was successful in 11 patients. There were no embolic complications.

\section{ANTICOAGULATION}

At the time of initial study 41 of the 100 patients had been treated with anticoagulants for at least one week, including 22 treated for at least one month. Fourteen of these 41 patients $(34 \%)$ were found to have left atrial or left ventricular thrombus by transoesophageal echocardiography compared with $4 / 59(7 \%)$ not receiving such treatment. Twenty $(45 \%)$ of these 41 patients had left atrial or left ventricular spontaneous echo contrast by transoesophageal echocardiography compared with $15 / 59(25 \%)$ not receiving such treatment.

\section{Discussion}

The management of cerebral ischaemic events is hindered by difficulty in clinically distinguishing cardiogenic embolism from other ischaemic events. ${ }^{1819}$ The clinical features of cardiogenic embolism can overlap with local artery-to-artery embolism, and about a third of patients found to have a potential cardiac source of embolism after a cerebral ischaemic event also have cerebrovascular atherosclerosis. ${ }^{1}$ Similar diagnosis difficulties arise with peripheral ischaemic events. ${ }^{3}$ Further, the detection of potential cardiac sources of embolism, particularly left atrial thrombi, is limited by the low sensitivity of current imaging techniques. Clinical and necropsy studies suggest that embolism from left atrial thrombi accounts for $50-70 \%$ of cerebral ischaemic events in patients with non-valvar atrial fibrillation. ${ }^{20}$ Non-valvar atrial fibrillation is the most common cardiac condition implicated in presumed embolic stroke, with a fivefold increase in stroke risk compared with sinus rhythm..$^{20}$

Although surgical series show that a large proportion of left atrial thrombi are located in the appendage, ${ }^{21}{ }^{22}$ this structure is difficult to image with transthoracic echocardiography and thrombi in the left atrial appendage are rarely detected by this method. ${ }^{21}{ }^{22}$ In contrast, transoesophageal echocardiography provides high frequency, close range imaging of the left atrium and appendage, which allows visualisation of thrombus, spontaneous contrast, and other abnormalities. ${ }^{810}$

Our results confirm the improved yield of potential sources of embolism with transoesophageal echocardiography, particularly for left atrial thrombi $(p<0.005)$ and left atrial spontaneous echo contrast $(p<0.0001)$. Twelve of the 13 left atrial thrombi were found in patients with atrial fibrillation and in the 53 patients in atrial fibrillation transoesophageal echocardiography showed potential embolic sources in 36 but transthoracic echocardiography showed them in only one ( $p<0.0001)$.

In our group of 63 patients referred after suspected cardiogenic embolism, we detected a potential embolic source by transoesophageal echocardiography in 27 patients $(43 \%)$ compared with three $(5 \%)$ (including one false positive) by transthoracic echocardiography $(p<0.0001)$. In 12 of these patients intracar- 
diac thrombus was detected only by transoesophageal echocardiography, and this resulted in warfarin treatment being started in two patients. The high yield of abnormal findings is related to our patient selection; we included patients with clinically suspected cardiogenic embolism and in whom transthoracic echocardiography alone was considered unlikely to detect potential embolic sources. In comparison, the incidence of thrombi detected by transoesophageal echocardiography in unselected patients with cerebral ischaemic events was $0-4 \% .{ }^{1123}$ Selection of patients for transoesophageal echocardiography should take into account both the likelihood of an abnormal finding and the potential impact on patient management.

Left atrial spontaneous echo contrast has recently attracted interest as a marker of thromboembolism. ${ }^{14}$ Earlier work suggested that spontaneous echo contrast is caused by aggregates of red cells in low flow states ${ }^{24} 25$ platelets too may also be involved. ${ }^{26}$ Most patients with left atrial thrombi also have left atrial spontaneous contrast; ${ }^{14}$ this was found in 10 of 13 patients in our series. Thus spontaneous echo contrast may prove to be a useful marker of sluggish blood flow-a condition favouring thrombus formation. In vitro data suggest that spontaneous echo contrast is not prevented by anticoagulant treatment. ${ }^{24}$ Indeed we found a higher incidence of both thrombi and spontaneous echo contrast in patients receiving warfarin, reflecting associated conditions such as atrial fibrillation, mitral valve replacement, and recent embolism.

We also studied patients before percutaneous balloon dilatation of the mitral valve and DC cardioversion of atrial fibrillation-both procedures with a risk of cardiogenic embolism. Half of the patients in our series with both atrial fibrillation and mitral stenosis were found to have previously unrecognised thrombi in the left atrial appendage; their discovery led to important changes in management. In addition, balloon dilatation of the mitral valve was performed without embolic complications in all patients without evidence of left atrial thrombus. We now perform transoesophageal echocardiography as a routine procedure before percutaneous balloon dilatation of the mitral valve.

There are no previous reports of the effect of balloon dilatation of the mitral valve on left atrial thrombi or spontaneous echo contrast, although disappearance of spontaneous contrast after mitral valve replacement has been reported. ${ }^{13}$ We found that left atrial spontaneous echo contrast disappeared in patients with the largest increase in mitral valve areathat is, a mitral valve area of at least $2 \mathrm{~cm}^{2}$. This is consistent with the reported association between left atrial spontaneous contrast and low left atrial flow rates. ${ }^{13}$

Successful balloon dilatation of the mitral valve also resulted in resolution of or reduction of left atrial appendage thrombi in three patients. However, one patient with atrial fibrillation and a mitral valve area of $1.5 \mathrm{~cm}^{2}$ who was not receiving warfarin developed a thrombus in the main left atrial cavity after balloon dilatation of the mitral valve.

Cardioversion of atrial fibrillation also carries a small risk of major systemic embolism, even with prior anticoagulation. ${ }^{5} \mathrm{~A}$ method of detecting left atrial thrombi or perhaps dense spontaneous echo contrast before cardioversion may allow the procedure to be timed more appropriately and the need for anticoagulation to be better assessed. In this series all patients in whom transoesophageal echocardiography did not show left atrial thrombus underwent cardioversion without embolic complications.

Our selection criteria limit extrapolation of our findings to all patients after stroke or transient ischaemic attack. Those who interpreted the transoesophageal echocardiographic results were not formally blinded to the transthoracic results. However, the studies were performed and reported independently and prospectively by separate experienced operators. Single plane transoesophageal echocardiography does not optimally visualise the left ventricular apex, which is in the far field of view, and small apical thrombi may be missed. Finally, there are few data on the correlation of echocardiographic and pathological findings for left atrial thrombi, although a preliminary report showed high sensitivity and specificity compared with surgical findings. ${ }^{27}$

Future indications for transoesophageal echocardiography may include the assessment of left atrial thrombi and spontaneous echo contrast in patients with non-valvar atrial fibrillation without previous embolism, in whom the risk of stroke is fivefold higher than in patients in sinus rhythm. ${ }^{20}$ There has been controversy about the role of long term anticoagulant therapy in these patients, though recent evidence suggests that warfarin treatment may be beneficial. ${ }^{28}$ Because anticoagulant therapy is associated with considerable morbidity and occasional mortality, it would be useful to be able to select patients at higher risk of thromboembolic complications. Transoesophageal echocardiography may provide a method of identifying these patients.

We conclude that transoesophageal echocardiography is a safe procedure that is more sensitive than transthoracic echocardiography for the detection of potential cardiac sources of embolism, particularly in patients with atrial fibrillation. Transoesophageal echocardiography is also useful in screening selected patients at risk of cardiogenic embolism. Further work is required regarding the prognostic implications of transoesophageal echocardiographic findings, such as spontaneous echo contrast, especially in patients with nonvalvar atrial fibrillation.

\footnotetext{
1 Cerebral Embolism Task Force. Cardiogenic Brain Embolism. Arch Neurol 1989;46:727-43.

2 Wolf PA Dawber TR, Thomas HE Kannel WB. Epideolf PA, Dawber TR, Thomas HE, Kannel WB. Epidemiologic assess stroke:

3 Dregelid EB, Stangeland LB, Eide GE, Trippestad A Characteristics of patients operated on because of suspected arterial embolism: a multivariate analysis. Surgery 1988;104:530-6.

4 Nishimura RA, Holmes DR Jr, Reeder GS. Percutaneous balloon valuloplasty. Mayo Clin Proc 1990;65:198-220.
} 
5 Mancini JGB, Goldberger AL. Cardioversion of atrial fibrillation: consideration of embolization, anticoagulafibrillation: consideration of embolization, anticoagulation, prophylactic pacema
Heart $J$ 1982;104:617-21.

6 Lovett JL, Sandok BA, Giulani ER, Nasser FN. Twodimensional echocardiography in patients with focal cerebral ischemia. Ann Intern Med 1981;95:1-4.

7 Knopman DS, Anderson DC, Asinger RW, Greenland P, Mikell F, Good DC. Indications for echocardiography in patients with ischemic stroke. Neurology 1982;32 1005-11.

8 Aschenberg W, Schluter M, Kremer P, Schroder E, Siglow $V$, Bleifeld $W$. Transoesophageal two-dimensional echocardiography for the detection of left atrial appendage thrombus. J Am Coll Cardiol 1986;7:163-6.

9 Zenker G, Erbel R, Kramer G, et al. Transoesophageal twodimensional echocardiography in young patients with cerebral ischemic events. Stroke 1988;19:345-8.

10 Daniel WG, Angermann C, Engberding R, et al. Transoesophageal echocardiography in patients with cerebral
ischemic events and arterial embolism-a European ischemic events and arterial embolism - a European II):II-473.

11 Pop G, Sutherland GR, Koudstaal PJ, Sit TW, de Jong G, Roelandt JRTC. Transoesophageal echocardiography in the detection of intracardiac embolic sources in patient with transient ischemic attacks. Stroke 1990;21:560-5.

12 Seward JB, Khandheria BK, Oh JK, et al. Transoesophageal echocardiography: technique, anatomic correlations, implementation and clinical applications. Mayo Clin Pro 1988;63:649-80.

13 Beppu S, Nimura Y, Sakakibara H, et al. Smoke-like echo in the left atrial cavity in mitral valve disease: Its features and significance. J Am Coll Cardiol 1985;6:744-9.

14 Daniel WG, Nellessen U, Schroder E, et al. Left atrial spontaneous echo contrast in mitral valve disease: an indicator for an increased thromboembolic risk. J Am Coll Cardiol 1988;11:1204-11.

15 Hatle L, Angelsen B, Tromsdal A. Non-invasive assessment of atrioventricular pressure half-time by Doppler of atrioventricular pressure half-time

16 Lechat P, Mas JL, Lascault G, et al. Prevalence of patent foramen ovale in patients with stroke. N Engl J Med 1988; 318:1148-52.
17 Nobuyoshi M, Hamasaki N, Kimura T, et al. Indications, complications, and short-term clinical outcome of percutaneous transvenous

18 Humphrey PRD, Harrison MJG. How often can an embolic stroke be diagnosed clinically? A clinicopathological stroke be diagnosed clinically? A clinicopat
correlation. Postgrad Med J 1985;61:1039-42.

19 Ramirez-Lassepas M, Cipolle RJ, Bjork RJ, et al. Can embolic stroke be diagnosed in the basis of neurologic embolic stroke be diagnosed in the basis

20 Sherman DG, Dyken ML, Fisher M, Harrison MJG, Hart RG. Cerebral embolism. Chest 1986;89(suppl 2): 82S-98S

21 Shrestha NK, Moreno FL, Narciso FV, Torres L, Calleja HB. Two-dimensional echocardiographic diagnosis of left atrial thrombus in rheumatic heart disease. A clinicopathologic study. Circulation 1983;67:341-7.

22 Bansal RC, Heywood JT, Applegate PM, Jutzy KR. Detection of left atrial thrombi by two-dimensional echocardiography and surgical correlation in 148 patients with mitral valve disease. Am J Cardiol 1989;64:243-6.

23 Jacobs NH, Whyte S, Kelleher AD, Juarez CC, Garrick R, Feneley MP. Limited role of transesophageal echocardiography in unselected patients with cerebral ischaemic diography in unselected patients with cerebral iI):III-110.

24 Siel $B$ ablu , blood durin

25 Mikell FL, Asinger RW, Elsperger KJ, Anderson WR Hodges $M$. Regional stasis of blood in the dysfunctional Hodges $M$. Regional stasis of blood in the dysfunctional left ventricle: echocardiographic detection and differentia-

26 Mahoney C, Sublett KL, Harrison MR. Resolution of Mahoney C, Sublett KL, Harrison MR. Resolution of
spontaneous contrast with platelet disaggregatory therapy spontaneous contrast with platelet disaggregatory
(trifluoperazine). Am J Cardiol 1989;63:1009-10

27 Matsumura M, Shah P, Kyo S, Omoto R. Advantages of transesophageal echo for correct diagnosis on small left atrial thrombi in mitral stenosis [abstract]. Circulation 1989;80(suppl II):II-678.

28 Petersen P, Boysen G, Godtfredsen J, Andersen ED Andersen B. Placebo-controlled, randomised trial of warfarin and aspirin for prevention of thromboembolic complications in chronic atrial fibrillation: The Copenhagen AFASAK study. Lancet 1989;i:175-9.

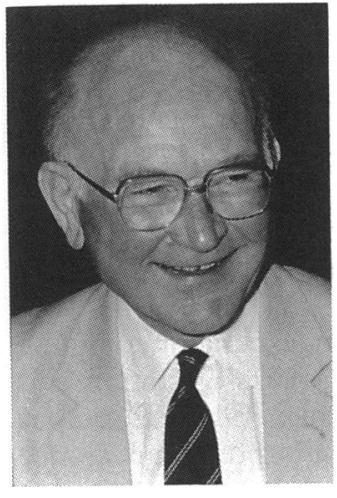

\section{EIRIAN WILLIAMS}

Bill Williams, MD, FRCP, died on 31 March, aged 65 , after a long struggle against an aggressive prostatic carcinoma. He spent 30 years as physician in general medicine and cardiology at Withybush Hospital, Haverfordwest, Pembrokeshire. He was a member of the Association of Physicians of Great Britain and Ireland and of the British Cardiac Society.

$\mathrm{He}$ was an international authority on brucellosis and published several papers on this topic in the Lancet in the 1970s. It was appropriate that he was asked to give the Donald Hunter Memorial Lecture to the Royal College of Physicians on this topic since Hunter was one of his mentors at the London Hospital and a lifelong friend.
Apart from being a dedicated and gifted clinician his main achievement was the creation of Withybush General Hospital in Haverfordwest, Pembrokeshire, which resulted out of his single-minded efforts. His contributions to postgraduate medicine in the area were recognised when the library in the postgraduate centre was named after him.

He will be remembered with affection by his many friends as an accomplished gardener, no mean wet fly fisher, and as a man who never gave up a cause-be it the developmment of his hospital or of academic cardiology in Cardiff. $\mathrm{He}$ is survived by his wife, three children, and seven grandchildren.

REG HALL 